\title{
Correction to: The Schwartz space of a smooth semi-algebraic stack
}

\author{
Yiannis Sakellaridis ${ }^{1,2}$ \\ Published online: 3 October 2018 \\ ○) Springer Nature Switzerland AG 2018
}

\section{Correction to: Sel. Math. New Ser. (2016) 22:2401-2490 https://doi.org/10.1007/s00029-016-0285-3}

The purpose of this note is to fix two gaps in the construction of Schwartz spaces of semi-algebraic stacks in [4], and to strengthen some statements, replacing quasiisomorphisms by homotopy equivalences. I am grateful to Avraham Aizenbud, Shachar Carmeli, and Dmitry Gourevitch for pointing out the gaps, and suggesting the stronger statements.

The first gap is in the proofs of Propositions 3.1.2 and 3.1.4, where I misquote [3, Theorem A.1.1] and write a Schwartz function as a product of two Schwartz functions. There is also an obvious typo in the statement of Proposition 3.1.4: the sequence appearing should end with $\stackrel{\partial_{0}}{\rightarrow} \mathcal{S}(Y) \rightarrow 0$. Moreover, with this gap corrected, a stronger statement is actually proven in these two propositions than claimed. Namely, the sequence of Proposition 3.1.4 (with the aforementioned typo corrected) is not just strictly exact, but homotopic to zero. I formulate this here as a proposition, which supersedes both of Propositions 3.1.2 and 3.1.4 in the paper, and indicate the corrections needed for a complete proof.

Proposition 1 Let $\pi: X \rightarrow Y$ be a smooth surjective morphism of Nash manifolds. Let $[X]_{Y}^{n}=$ the fiber product of $n$ copies of $X$ over $Y$ (whose projection map to $Y$ is still denoted by $\pi$ ), and consider the complex

$$
\left(\mathcal{S}\left([X]_{Y}^{n}\right)\right)_{n}: \cdots \rightarrow \mathcal{S}\left([X]_{Y}^{3}\right) \rightarrow \mathcal{S}\left([X]_{Y}^{2}\right) \rightarrow \mathcal{S}(X) \rightarrow 0
$$

The original article can be found online at https://doi.org/10.1007/s00029-016-0285-3.

$\bowtie \quad$ Yiannis Sakellaridis

sakellar@rutgers.edu

1 Department of Mathematics and Computer Science, Rutgers University - Newark, 101 Warren Street, Smith Hall 216, Newark, NJ 07102, USA

2 Department of Mathematics, School of Applied Mathematical and Physical Sciences, National Technical University of Athens, Heroon Polytechneiou 9, 15780 Zografou, Greece 
with differentials $\partial_{n}: \mathcal{S}\left([X]_{Y}^{n+1}\right) \rightarrow \mathcal{S}\left([X]_{Y}^{n}\right)$ induced from the alternating sum of push-forwards when a copy of $X$ is deleted, as in [4, Proposition 3.1.4]. Consider $\mathcal{S}(Y)$ as a complex in degree zero, and the morphism of complexes

$$
\pi_{!}:\left(\mathcal{S}\left([X]_{Y}^{n}\right)\right)_{n} \rightarrow \mathcal{S}(Y)
$$

induced by the push-forward $\pi_{!}: \mathcal{S}(X) \rightarrow \mathcal{S}(Y)$. This morphism is a homotopy equivalence.

Proof [3, Theorem A.1.1] states that any $f \in \mathcal{S}\left([X]_{Y}^{n}\right)$ can be written as a finite sum

$$
f(\mathbf{x})=\sum_{i=1}^{m} \phi_{i}(\pi(\mathbf{x})) f_{i}(\mathbf{x}),
$$

where $\phi_{i}$ is a Schwartz function on $Y$ and $f_{i} \in \mathcal{S}\left([X]_{Y}^{n}\right)$. Over an Archimedean field it is not true, in general, that it can be written as a product $\phi \cdot f_{0}$, as claimed in [4].

But now, assuming, as in the proofs of [4, Propositions 3.1.2 and 3.1.4], that the differential $\partial_{n-1} f \in \mathcal{S}\left([X]_{Y}^{n-1}\right)$ vanishes, an extra complication arises, because only the sum $\sum_{i=1}^{m} \phi_{i}(\pi(\mathbf{x})) \partial_{n-1} f_{i}(\mathbf{x})$ vanishes, not each term $\partial_{n-1} f_{i}$ individually. The next step in the proofs is to disintegrate $\phi$ to an element $h$ of some space of "relative Schwartz measures" $\mathcal{S}^{\prime}(X)$ (whose push-forwards to $Y$ are Schwartz functions-see the proof of [4, Proposition 3.1.2] for details). For the argument to go through as stated, we need to do this compatibly for all $\phi_{i}$ 's. Namely, let us assume that the base field is $F=\mathbb{R}$ (because in the non-Archimedean case there is no issue, and in the complex case we may work by restriction of scalars over $\mathbb{R}$ without changing the final statement).

Let us first discuss the special case where the morphism $X \rightarrow Y$ admits a Nash section $\sigma: Y \rightarrow X$, which, in addition, extends to a tubular neighborhood $\iota:$ $Y \times B_{r} \hookrightarrow X$, where $r$ is the relative dimension of the map $\pi$, and $B_{r}$ is the open unit ball in $\mathbb{R}^{r}$. Then, choosing a Schwartz measure $\mu \in \mathcal{S}\left(B_{r}\right)$ with total mass 1 , we can set $h_{i}:=\iota_{!}\left(\phi_{i} \otimes \mu\right) \in \mathcal{S}^{\prime}(X)$. Then $\pi_{!} h_{i}=\phi_{i}$, and $\sum_{i} h_{i}\left(x_{0}\right) \partial_{n-1} f_{i}\left(x_{1}, \ldots, x_{n-1}\right)=0$; the proofs of the two Propositions now go through as stated. Moreover, the tubular neighborhood gives rise to an embedding, again to be denoted by the same letter:

$$
\iota:[X]_{Y}^{n} \times B_{r} \hookrightarrow[X]_{Y}^{n+1}
$$

(with $B_{r}$ determining the last coordinate), and a choice of $\mu$ as above allows us to define linear maps

$$
H_{n}: \mathcal{S}\left([X]_{Y}^{n}\right) \rightarrow \mathcal{S}\left([X]_{Y}^{n+1}\right)
$$

by $H_{n}(f)=\iota !(\mu \otimes f)$. This includes the case of $H_{0}: \mathcal{S}(Y) \rightarrow \mathcal{S}(X)$, which is a section for the push-forward map. One then easily checks that $H_{n}$ is a homotopy between $H_{0} \circ \pi$ ! and the identity on the complex $\left(\mathcal{S}\left([X]_{Y}^{n}\right)\right)_{n}$; in other words, $\pi_{\text {! : }}$ $\left(\mathcal{S}\left([X]_{Y}^{n}\right)\right)_{n} \rightarrow \mathcal{S}(Y)$ is a homotopy equivalence. 
We have up to now assumed that the morphism $X \rightarrow Y$ admitted a section with a tubular neighborhood. Such tubular neighborhoods exist locally over $Y$ [2, 2.4.3], [1, Theorem 3.6.2]. The last step to correct the proof is to show that all statements are local over $Y$ (in the semi-algebraic topology). For this, given a (finite) semi-algebraic open cover $Y=\cup_{j} Y_{j}$, we use the "Schwartz partition of unity" of [1, Theorem 4.4.1], which is a collection of tempered functions $u_{j}$, with $u_{j}$ supported on $Y_{j}, \sum_{j} u_{j}=1$, and the property that multiplication by $u_{j}$ turns a Schwartz function (or measure) on $Y$ to a Schwartz function (or measure) on $Y_{j}$. Multiplication by such a partition of unity gives, in the usual way, a homotopy equivalence between $\mathcal{S}(Y)$ and the Čech complex of Schwartz spaces associated to the cover, and similarly for $\left(\mathcal{S}\left([X]_{Y}^{n}\right)\right)_{n}$ and the total complex of the associated Čech bicomplex. Of course, multiplication by $u_{j} \circ \pi$ will not change the property $\partial_{n-1} f=0$ (of $f \in \mathcal{S}\left([X]_{Y}^{n}\right)$ ), so we are reduced to the case where a section with a tubular neighborhood exists.

The second gap is in the proof of functoriality in Theorem 3.3.1. Again, once the gap is fixed, a stronger statement is actually proven:

Theorem 2 Let $\mathfrak{X}$ be a Nash stack. For any two presentations $X_{1} \rightarrow \mathfrak{X}, X_{2} \rightarrow \mathfrak{X}$, the Schwartz complexes

$$
\left(\mathcal{S}\left(\left[X_{i}\right]_{\mathfrak{X}}^{n}\right)\right)_{n}: \cdots \rightarrow \mathcal{S}\left(\left[X_{i}\right]_{\mathfrak{X}}^{3}\right) \rightarrow \mathcal{S}\left(\left[X_{i}\right]_{\mathfrak{X}}^{2}\right) \rightarrow \mathcal{S}\left(X_{i}\right) \rightarrow 0
$$

$(i=1,2)$ are canonically homotopy equivalent, and hence can be denoted by $\mathcal{S}_{\bullet}(\mathfrak{X})$.

The association $\mathfrak{X} \mapsto \mathcal{S}_{\bullet}(\mathfrak{X})$ is functorial with respect to smooth 1-morphisms of Nash stacks, up to homotopy.

Proof For notational simplicity, let us in the proof denote $X_{1}$ by $X, X_{2}$ by $Y$, and $X^{(i)} Y^{(j)}:=[X]_{\mathfrak{X}}^{i} \times \mathfrak{X}[Y]_{\mathfrak{X}}^{j}$. We also denote $X^{(1)} Y^{(1)}$ by $R_{X Y}, X^{(2)}$ by $R_{X}$, and $Y^{(2)}$ by $R_{Y}$; hence, for $i, j \geq 1$ we have

$$
X^{(i)} Y^{(j)}=\left[R_{X}\right]_{X}^{i-1} \times_{X} R_{X Y} \times{ }_{Y}\left[R_{Y}\right]_{Y}^{j-1} .
$$

This is a unique Nash manifold up to unique isomorphism, once the Nash manifolds $R_{X Y}, R_{X}, R_{Y}$ (with their morphisms to $X, Y$ ) have been fixed. The "presentations" $X \rightarrow \mathfrak{X}, Y \rightarrow \mathfrak{X}$ implicitly include the groupoids $R_{X} \rightrightarrows X, R_{Y} \rightrightarrows Y$, but the gap in the proof of Theorem 3.3.1 is that it is not taken into account that $R_{X Y}$ is only defined up to automorphisms over $X \times Y$. Thus, we need to make sure that, in the proof of Theorem 3.3.1, composition with automorphisms $\tau: R_{X Y} \rightarrow R_{X Y}$ over $X \times Y$ does not change the homotopy class of the equivalence $\left(\mathcal{S}\left(X^{(n)}\right)\right)_{n} \stackrel{\sim}{\rightarrow}\left(\mathcal{S}\left(Y^{(n)}\right)\right)_{n}$.

We revisit the proof, in order also to explain that it can be strengthened to a homotopy equivalence. The essential statement is that, if we consider the total complex $\mathbb{T}_{X Y}$ associated to the bicomplex $\left(\mathcal{S}\left(X^{(i)} Y^{(j)}\right)\right)_{i, j \geq 1}$, its natural push-forward maps to the complexes $\left(\mathcal{S}\left(X^{(i)}\right)\right)_{i \geq 1},\left(\mathcal{S}\left(Y^{(j)}\right)\right)_{j \geq 1}$ are homotopy equivalences.

Let us briefly see why: Applying Proposition 1 above, for any $i$, the natural pushforward

$$
\left(\mathcal{S}\left(X^{(i)} Y^{(j)}\right)\right)_{j} \longrightarrow\left(0 \rightarrow \mathcal{S}\left(X^{(i)}\right) \rightarrow 0\right)
$$


is a homotopy equivalence. The construction of a homotopy inverse relied on choosing, locally, a section with a tubular neighborhood:

$$
X^{(i)} \times B_{r} \hookrightarrow X^{(i)} Y^{(1)} .
$$

In our setting, we can choose once and for all a section with a tubular neighborhood

$$
\iota: X \times B_{r} \hookrightarrow R_{X Y},
$$

at least locally on $X$. (It is clearly enough to work locally over $X$ here, in order to prove the homotopy equivalence of the total complex with $\left(\mathcal{S}\left(X^{(i)}\right)\right)_{i}$.) This induces sections

$$
X^{(i)} \times B_{r}=\left[R_{X}\right]_{X}^{(i-1)} \times_{X} X \times B_{r} \hookrightarrow X^{(i)} Y^{(1)}=\left[R_{X}\right]_{X}^{(i-1)} \times_{X} R_{X Y}
$$

for all $i \geq 1$; the resulting homotopy inverses constructed in the proof of Proposition 1 (denoted by $H_{0}$ there) will now be, by construction, chain maps of complexes:

$$
H_{0}:\left(\mathcal{S}\left(X^{(i)}\right)\right)_{i \geq 1} \rightarrow\left(\mathcal{S}\left(X^{(i)} Y^{(1)}\right)\right)_{i \geq 1} \hookrightarrow \mathbb{T}_{X Y}
$$

Thus, the statement of Proposition 1 extends to the morphism of complexes $\mathbb{T}_{X Y} \rightarrow$ $\left(\mathcal{S}\left(X^{(i)}\right)_{i \geq 1}\right.$, and shows that it is a homotopy equivalence.

Let us now assume that $\tau: R_{X Y} \rightarrow R_{X Y}$ is an automorphism over $X \times Y$; by (1), it induces automorphisms $\tau_{\text {! }}$ of all Schwartz spaces $\mathcal{S}\left(X^{(i)} Y^{(j)}\right), i, j \geq 1$. I claim that the composition $\tau_{!} \circ H_{0}$ is still a homotopy inverse to the push-forward map $p_{X}: \mathbb{T}_{X Y} \rightarrow\left(\mathcal{S}\left(X^{(i)}\right)\right)_{i \geq 1}$. Indeed, in the construction of this homotopy inverse, $\tau$ just modifies the tubular neighborhood (2), that is, $\tau_{!} H_{0}$ is obtained by the same construction, using the tubular neighborhood $\tau \circ \iota$. Thus, it is homotopy inverse to the canonical push-forward map $p_{X}$.

But $\tau_{!} \circ H_{0}$ is also homotopy inverse to the composition $p_{X} \circ \tau_{!}^{-1}$. We deduce that the morphisms $p_{X}$ and $p_{X} \circ \tau_{!}^{-1}$ are homotopic. The same holds for the analogous morphisms $p_{Y}$ and $p_{Y} \circ \tau_{!}^{-1}$; thus, the homotopy class of the equivalence of Schwartz complexes

$$
\left(\mathcal{S}\left(X^{(i)}\right)\right)_{i \geq 1} \stackrel{\sim}{\rightarrow}\left(\mathcal{S}\left(Y^{(j)}\right)\right)_{j \geq 1}
$$

is fixed under automorphisms of $X \times \mathfrak{X} Y$.

\section{References}

1. Aizenbud, A., Gourevitch, D.: Schwartz functions on Nash manifolds. Int. Math. Res. Not. IMRN (5):Art. ID rnm 155, 37. https://doi.org/10.1093/imrn/rnm155 (2008)

2. Aizenbud, A., Gourevitch, D.: The de-Rham theorem and Shapiro lemma for Schwartz function on Nash manifolds. Isr. J. Math. 177, 155-188 (2010). https://doi.org/10.1007/s11856-010-0042-9 
3. Aizenbud, A., Gourevitch, D.: Smooth transfer of Kloosterman integrals (the Archimedean case). Am. J. Math. 135(1), 143-182 (2013). https://doi.org/10.1353/ajm.2013.0000

4. Sakellaridis, Y.: The Schwartz space of a smooth semi-algebraic stack. Sel. Math. (N.S.) 22(4), 24012490 (2016). https://doi.org/10.1007/s00029-016-0285-3 